\title{
NOMA-2000 vs. PD-NOMA: An Outage Probability Comparison
}

\author{
İsmail Coşandal, Student Member, IEEE, Mutlu Koca, Senior Member, IEEE, Ezio Biglieri, Life Fellow, IEEE and \\ Hikmet Sari, Life Fellow, IEEE
}

\begin{abstract}
Non-orthogonal multiple access (NOMA) is an emerging technique promising to accommodate the increasing data rate requirements of next generation networks. In powerdomain (PD) NOMA, this challenge is addressed by allocating two or more users on each orthogonal resource block while assigning significantly different power levels to all users. Recently, an interesting alternative to conventional PD-NOMA has been NOMA-2000 where multiple access is done using two sets of orthogonal waveforms. In this paper we make a comparison of these two NOMA schemes based on their outage probabilities where the analytical expressions are derived without any simplifying assumption about the statistical dependencies caused by the presence of more than one user signal in each resource block. These derivations are validated by computer simulation results, which appear in tight agreement with the analysis and corroborate the claim that NOMA-2000 may significantly outperform PD-NOMA under realistic channel conditions and with different overload factors.
\end{abstract}

Index Terms-Non-orthogonal multiple access, outage probability, performance evaluation.

\section{INTRODUCTION}

Non-orthogonal multiple access (NOMA) is based on signal superposition at the transmitter side and successive interference cancellation (SIC) at the receiver. Signal superposition can be achieved by using different power levels, modulation types, non-orthogonal codebooks, etc., as summarized in [1], [2]. The most popular version on which the literature has been heavily based on is the so-called power-domain NOMA (PDNOMA). In its simplest form with two users this consists of superposing a strong user signal and a weak user signal. At the receiver, each user message is decoded from the received messages in a sequence depending on the channel gains and also using the SIC procedure to cancel the interference from the other user(s) as shown in [3].

Although it is relatively easy to implement and known to potentially achieve improved rates, the PD-NOMA approach faces two major practical problems: First, while a uniform power distribution among users seems to be the natural choice for providing them with the same data rate and performance, PD-NOMA needs signals in the same resource block to have different power levels at the receiver. This means that

İ. Coşandal and M. Koca are with the Department of Electrical and Electronics Engineering, Boğaziçi University, Bebek 34342 Istanbul, Turkey, E-mails: \{ismail.cosandal, mutlu.koca\} @boun.edu.tr. Their research was supported by TUBITAK with the project $119 \mathrm{~N} 154$.

E. Biglieri is with the Departament TIC, Universitat Pompeu Fabra, 08008 Barcelona, Spain, E-mail:e.biglieri@ieee.org. His work was supported by the H2020 Framework Programme/ERC grant 694974.

H. Sari is with Nanjing University of Posts and Telecommunications, 66 Xinmofan Road, Gulou District, Nanjing, 210003 China and with Sequans Communications, Les Portes de la Défense, 15-55 Boulevard Charles de Gaulle, 92700 Colombes, France, E-mail:hsari@ieee.org. providing the same quality-of-service (QoS) for users with superimposed signals may require the use of explicit power allocation or resource scheduling strategies at the expense of imposing additional computational constraints on the system. Second, since user signals interfering with each other are decoded by a single-shot SIC receiver, a signal-to-noise ratio (SNR) degradation occurs at practical bit-error rate (BER) values, which might be significant unless the interfering signal power is substantially reduced.

To compensate for these drawbacks, an alternative NOMA technique was recently proposed in [4]-[6], as a revival of an early NOMA concept introduced in [7], [8]. This approach, called NOMA-2000, consists of superimposing signals from two user groups rather than from two single users. In a multicarrier system with $N$ subcarriers, NOMA-2000 is employed as follows: A first group of $N$ active users transmit in orthogonal frequency-division multiple access (OFDMA) mode with a separate subcarrier assigned to each user. Next, a second group of $M \leq N$ active users transmit in multi-carrier code-division multiple access (MC-CDMA) mode by spreading their signals over the entire band and superimposing them to the signals of the first group of users. Notice that, with this approach, the power imbalance between user signals at the receiver is inherent in the spreading of the MC-CDMA signals, and hence the power imbalance requirement of PD-NOMA disappears. Another virtue of this approach is that the iterative interference cancellation used at the receiver reduces the SNR degradation virtually to zero, which is not possible with the single-shot SIC receivers used in PD-NOMA. Bit-error rate performance of NOMA-2000 is compared with that of conventional PDNOMA over Gaussian channels in [4], [5] and over fading channels in [6] both with a static channel assignment and also with a dynamic user grouping strategy. In almost all scenarios NOMA-2000 is shown to significantly outperform PD-NOMA. Notice that compared to PD-NOMA, NOMA-2000 has a small increase in the transceiver circuitry and the computational complexity due mainly to the additional spreading/despreading operations required to decode the signals of the MC-CDMA users, which is nevertheless justified by the substantial performance gains mentioned above.

In this paper, we compare PD-NOMA and NOMA-2000 in terms of their outage probability, which is the relevant performance metric when coding is used and the channel is subject to non-ergodic fading. We consider an uplink multicarrier communication system employing either PD-NOMA on each of its subcarriers or NOMA-2000 with two sets of orthogonal waveforms, and derive the exact outage probabilities over Rayleigh fading channels. For clarity of presentation and ease in derivations we consider the use of a practical but 
effective user grouping strategy given in [6], however the analytical framework presented here can also be extended to the configurations with more sophisticated user grouping strategies. The outage probability of PD-NOMA was analyzed, e.g., in [9]-[12] with the simplifying assumption that the outage events of different users occur independently. Notice that this assumption does not comply with the system or outage event model, and hence may lead to severely inaccurate results. On the other hand in [13], this dependency is taken into account but the outage probability derivations are made with a simple model where only single carrier PD-NOMA is studied with two users and the multicarrier scenario is not considered. For this reason, the first contribution of our work is to remove the independence assumption that appears in [9]-[12] and extend the analysis in [13] to the multicarrier PD-NOMA systems and to provide a theoretical framework incorporating the correlation among multiple user signals on each subcarrier. As a second contribution, we consider the same framework and the analytical outage probability expressions for NOMA-2000 as well. Then we compare the outage probability performances of PD-NOMA and NOMA-2000 via both exact analytical expressions and simulation results, which reveal that NOMA2000 significantly outperforms PD-NOMA in all scenarios with different overloading factors.

The rest of the paper is organized as follows. In Section II, we present the system models for both NOMA schemes. In Section III, the outage probabilities are defined and analytical calculations are derived. Simulation results are presented in Section IV followed by our conclusions in Section V.

\section{SySTEM MODEL}

In this section we present the basic principles and system models for the two NOMA systems in consideration. For both PD-NOMA and NOMA-2000, we consider an uplink multicarrier system model with $N$ subcarriers and $K>N$ users. We define $M=K-N$ as the number of excess users and the overloading factor (OF) as the ratio $\frac{M}{N}$ of the excess users to the total number of subcarriers. The total number of users can be partitioned into two groups, $U_{1}$ with users indexed as $1, \ldots, N$ and $U_{2}$ with the remaining $M$ overloading users, indexed as $N+1, \ldots, N+M$.

\section{A. PD-NOMA}

In PD-NOMA, we assume that each subcarrier is either assigned to a single user from $U_{1}$ or to two users, one from $U_{1}$ and the other from $U_{2}$. To distinguish between these two cases, we further partition $U_{1}$ into two subgroups $\hat{U}_{1}$ and $\tilde{U}_{1}$, where $\tilde{U}_{1}$ includes the users not sharing the subcarrier. We adopt the dynamic user grouping strategy in [6] where the users with the $N$ highest channel gains are assigned to the group $U_{1}$, while the remaining $M$ users with the lowest channel gains are assigned to the group $U_{2}$.

If users $n \in \hat{U}_{1}$ and $N+n \in U_{2}$ are on subcarrier $n$, the superimposed signal $r_{n}$ received on this subcarrier becomes

$r_{n}=\sqrt{P_{n}} h_{n} a_{n}+\sqrt{P_{N+n}} h_{N+n} b_{n}+z_{n}, \quad n \in\{1, \ldots M\}$

where $P_{n}$ and $P_{n+N}$ are the powers of the two users on subcarrier $n, h_{n}$ and $h_{n+N}$ are their channel coefficients, $a_{n}$ and $b_{n}$ are the information symbols transmitted by these two users, and $z_{n}$ is an additive white Gaussian noise (AWGN) term with variance $\sigma^{2}$. Each channel coefficient can be expressed as $h_{i}=g_{i} / l_{i}$ where $l_{i}$ is the large-scale path loss and $g_{i}$ is the small-scale channel fading coefficient that is assumed to be Rayleigh distributed. Therefore, the "channel gain" $V \triangleq\left|g_{i}\right|^{2}$ is an exponential random variable with the probability density function (PDF), i.e., $V \sim \operatorname{Exp}(\mu)$ where $\mu=\sigma^{2}$.

The BS decodes users' signals in decreasing power order by treating interfering signals as noises. After the first signal is decoded, the interference affecting the first-user signal is subtracted from the superimposed signal before the detection for the second-user signal starts in the SIC operation. The corresponding signal-to-interference plus noise ratio (SINR) for the first group of users (strong signal users) and the SNR for the second group of users (weak signal users) at each subcarrier $n(n \in\{1 \ldots M\})$ can be written as

$$
\Gamma_{U_{1}}^{(n)}=\frac{P_{n}\left|h_{n}\right|^{2}}{P_{N+n}\left|h_{N+n}\right|^{2}+\sigma^{2}}, \quad \Gamma_{U_{2}}^{(n)}=\frac{P_{N+n}\left|h_{N+n}\right|^{2}}{\sigma^{2}} .
$$

On the other hand, if user $k$ is the single user in a subcarrier, the received signal $r_{k}$ can be expressed as

$$
r_{k}=\sqrt{P_{k}} h_{k} a_{k}+z_{k}, \quad k \in\{M+1, \ldots, N\},
$$

and the corresponding SNR is $\Gamma_{k}=\frac{P_{k}\left|h_{k}\right|^{2}}{\sigma^{2}}$.

In conventional communication systems, user powers are determined so as to also compensate for the effect of path loss [14]. Specifically, as shown in [9], the power assignment to user $i$ can be done to generate a power imbalance such that

$$
P_{i}= \begin{cases}\min \left(P_{\max }, P_{\mathrm{t}} l_{i}^{2}\right), & i \in\{1 \ldots N\}, \\ \min \left(P_{\max }, \epsilon P_{\mathrm{t}} l_{i}^{2}\right), & i \in\{N+1 \ldots N+M\},\end{cases}
$$

where $P_{\max }$ is the maximum allowed power, $P_{\mathrm{t}}$ is the target received power and $\epsilon \in(0,1)$ is a system parameter that determines the amount of power imbalance. This enables outage probability to rely mainly on small scale fading coefficients.

\section{B. NOMA-2000}

Following [4]-[6], in uplink NOMA-2000 two sets of orthogonal signal waveforms are utilized: while OFDMA forms the first signal set, and MC-CDMA is used as the second signal set. The first $N$ users operate in OFDMA mode with one subcarrier assigned to each of them, while the additional $M$ users are assigned to one MC-CDMA waveform by spreading their signals using a length- $N$ Walsh-Hadamard $(\mathrm{WH})$ code. As with PD-NOMA, we assume that users are grouped according to the method described in [6]. The corresponding signal on subcarrier $n(n=1,2, \ldots, N)$ can be written as

$$
r_{n}=\sqrt{P_{n}} h_{n} a_{n}+I_{n}+z_{n} .
$$

Here $I_{n}$ denotes the interference on subcarrier $n$, given by

$$
I_{n}=\frac{1}{\sqrt{N}} \sum_{i=1}^{M} \sqrt{P_{N+i}}\left(w_{i, n} h_{N+i}\right) b_{i},
$$

where $w_{m, n}$ is the $n$th chip of the WH sequence associated with user $m$. The factor $1 / \sqrt{N}$ is included for energy normalization. Because of spreading, MC-CDMA users interfere less with OFDMA users, and the OFDMA user signals can be 
decoded under this interference. After that, the decoded signal $\hat{a}_{n}, n \in U_{1}$, is subtracted from the superimposed signal. Using multi-user MC-CDMA detection, the signal of the $m$ th MCCDMA user can be decoded from signal

$$
r_{m}=\sqrt{P_{N+m}} h_{N+m} b_{m}+I_{m}+u_{m},
$$

where $u_{m}$ is an AWGN term with the same statistics as $z_{n}$. $I_{m}$ is the interference caused by the spreading of the OFDMA signals, and can be expressed as

$$
I_{m}=\frac{1}{\sqrt{N}} \sum_{j=1}^{N} \sqrt{P_{j}}\left(w_{j, m} h_{j}\right)\left(a_{j}-\hat{a}_{j}\right) .
$$

The final SINR value of the $n$th OFDMA user and the SNR of the $m$ th MC-CDMA user can be written respectively as

$$
\Gamma_{n}=\frac{P_{n}\left|h_{n}\right|^{2}}{\frac{1}{N} \sum_{i=1}^{M} P_{i+N}\left|h_{i+N}\right|^{2}+\sigma^{2}}, \quad \Gamma_{m}=\frac{P_{m}\left|h_{m}\right|^{2}}{\sigma^{2}} .
$$

\section{Outage Probability Derivations}

In this section we define the outage events and derive the outage probabilities for NOMA users. We start with the simplest case of a single carrier and two users, next we extend our calculations to multicarrier NOMA.

\section{A. PD-NOMA}

Based on the system model of Section II in which user $n \in \hat{U}_{1}$ and user $N+n \in U_{2}$ share the same subcarrier, $E_{n}^{P D}$ and $E_{N+n}^{P D}$ are defined as the coverage events where user $n$ has an SINR level higher than the target SINR and user $N+n$ has an SNR level higher than a target SNR after SIC, respectively. The outage of user $n$ is defined when $E_{n}^{P D}$ does not occur. On the other hand, user $N+n$ is in outage if neither $E_{n}^{P D}$ nor $E_{N+n}^{P D}$ occurs, in which case the BS cannot decode the signal of user $N+n$ because it fails to decode the signal of user $n$.

The corresponding events can be written as

$$
\begin{aligned}
E_{n}^{P D} & =\left\{\frac{P_{n}\left|h_{n}\right|^{2}}{P_{N+n}\left|h_{N+n}\right|^{2}+\sigma^{2}} \geq \phi\right\} \\
& =\left\{\left|g_{n}\right|^{2} \geq \phi \frac{P_{N+n} l_{n}^{2}}{P_{n} l_{N+n}^{2}}\left|g_{N+n}\right|^{2}+\beta_{1}\right\} \\
& =\left\{\left|g_{n}\right|^{2} \geq \alpha\left|g_{N+n}\right|^{2}+\beta_{1}\right\}, \\
E_{N+n}^{P D} & =\left\{\frac{P_{N+n}\left|h_{N+n}\right|^{2}}{\sigma^{2}} \geq \phi\right\}=\left\{\left|g_{N+n}\right|^{2} \geq \beta_{2}\right\}(11) \\
E_{n}^{P D} \cap E_{N+n}^{P D} & =\left\{\left|g_{n}\right|^{2} \geq \alpha\left|g_{N+n}\right|^{2}+\beta_{1},\left|g_{N+n}\right|^{2} \geq \beta_{2}\right\},(12)
\end{aligned}
$$

where $\beta_{1} \triangleq \frac{\phi}{P_{t}} \sigma^{2}, \beta_{2} \triangleq \frac{\phi}{\epsilon P_{t}} \sigma^{2}, \alpha \triangleq \phi \epsilon$, and $\phi \triangleq\left(2^{\hat{R}}-1\right)$ and $\hat{R}$ is the target rate. Then the outage probabilities of users $n$ and $N+n$ can be formulated as

$$
\begin{aligned}
\operatorname{Pr}_{n, \text { out }} & =1-\operatorname{Pr}\left(E_{n}^{P D}\right), \\
\operatorname{Pr}_{N+n, \text { out }} & =1-\operatorname{Pr}\left(E_{n}^{P D} \cap E_{N+n}^{P D}\right),
\end{aligned}
$$

In some works (e.g., [9]-[12]), events $E_{n}^{P D}$ and $E_{N+n}^{P D}$ are assumed to be independent, which leads to $P r_{\mathrm{out}, \mathrm{N}+\mathrm{n}}=$
$1-\operatorname{Pr}\left(E_{n}^{P D}\right) \operatorname{Pr}\left(E_{N+n}^{P D}\right)$. However, it can be seen that the random variable $\left|g_{N+n}\right|^{2}$ is common to both events, and therefore these are generally not independent.

Starting with the simplest case where $N=1$ and $K=2$, outage probabilities of the events $E_{1}^{P D} E_{2}^{P D}$ and $E_{1}^{P D} \cap E_{2}^{P D}$ can be computed in closed form, using (10), (12) and (13), as

$$
\begin{aligned}
\operatorname{Pr}\left(E_{1}^{P D}\right) & =2 \int_{0}^{\infty} \int_{\alpha x_{2}+\beta_{1}}^{\infty} f_{V_{1}}\left(v_{1}\right) f_{V_{2}}\left(v_{2}\right) d v_{1} d v_{2} \\
& =\frac{2}{\alpha+1} \exp \left(-\beta_{1} / 2 \mu^{2}\right), \\
\operatorname{Pr}\left(E_{2}^{P D}\right) & =\int_{\beta_{2}}^{\infty} 2 f_{V}(v)\left(1-F_{V}(v)\right) d v=\exp \left(-\beta_{2} / \mu^{2}\right) \\
\operatorname{Pr}\left(E_{1}^{P D} \cap E_{2}^{P D}\right) & =2 \int_{\beta_{2}}^{\infty} \int_{\alpha v_{2}+\beta_{1}}^{\infty} f_{V_{1}}\left(v_{1}\right) f_{V_{2}}\left(v_{2}\right) d v_{1} d v_{2} \\
& =\frac{2}{\alpha+1} \exp \left\{-\frac{(\alpha+1) \beta_{2}}{2 \mu^{2}}\right\} \exp \left(-\beta_{1} / 2 \mu^{2}\right)
\end{aligned}
$$

Notice that the independence assumption is only valid when $\alpha=1$, which makes $\operatorname{Pr}\left(E_{1}^{P D} \cap E_{2}^{P D}\right)=$ $\operatorname{Pr}\left(E_{2}^{P D}\right) \operatorname{Pr}\left(E_{1}^{P D}\right)$.

Next we consider the general case of $N$ subcarriers and $K$ users. First, define two new random variables $X$ and $Y$ modeling the channel gains of the users in groups $U_{1}$ and $U_{2}$, respectively. We can derive the PDF of these two groups by assuming that any user in $U_{1}$ has a channel gain higher than a threshold value $\tau$, and vice versa for $U_{2}$. Manipulation of the original exponential distribution allows us to express the PDFs of $X$ and $Y$ as $f_{X}(x)=\frac{C_{1}}{2 \mu^{2}} \exp \left(-\frac{x}{2 \mu^{2}}\right),(x \geq \tau)$, and $f_{Y}(y)=\frac{C_{2}}{2 \mu^{2}} \exp \left(-\frac{y}{2 \mu^{2}}\right),(\tau>y \geq 0)$, where $C_{1}$ and $C_{2}$ are PDF normalization constants The threshold $\tau$ can be easily calculated as $\tau=2 \mu^{2} \ln (1+M / N)$.

The outage probability of users who share their subcarriers with an excess user, denoted $\operatorname{Pr}_{\hat{U}_{1} \text {,out }}^{P D}$, can be found as

$$
\begin{aligned}
& \operatorname{Pr}_{\hat{U}_{1}, \text { out }}^{P D}=1-\operatorname{Pr}\left(\left|g_{n}\right|^{2}>\alpha\left|g_{m}\right|^{2}+\left.\beta_{1}|| g_{n}\right|^{2}>\tau,\left|g_{m}\right|^{2}<\tau\right) \\
& \quad=1-C_{2} \int_{0}^{t_{1}} f_{Y}(y) d x-C_{2} C_{1} \int_{t_{1}}^{\tau} \int_{\alpha y+\beta_{1}}^{\infty} f_{X}(x) f_{Y}(y) d x d y \\
& \quad=1-\rho_{1} C_{2}\left(1-e^{-\frac{\tau-\beta_{1}}{2 \mu^{2} \alpha}}\right)-\gamma e^{-\frac{\beta_{1}}{2 \mu^{2}}}\left(e^{-\frac{(\alpha+1) t_{1}}{2 \mu^{2}}}-e^{-\frac{(\alpha+1) \tau}{2 \mu^{2}}}\right),
\end{aligned}
$$

where $\gamma \triangleq \frac{C_{1} C_{2}}{\alpha+1}$. The integral is divided into two parts at $t_{1} \triangleq \max \left(0, \frac{\tau-\beta_{1}}{\alpha}\right)$ because when the secondary users have channel gains lower than $\left(\tau-\beta_{1}\right) / \alpha$ the primary users on the same subcarrier are never in outage. Additionally, the outage probability for the users who are the sole dwellers of their respective subcarriers, denoted $\operatorname{Pr}_{\tilde{U}_{1} \text {, out }}^{P D}$, can be derived as

$$
\begin{aligned}
\operatorname{Pr}_{\tilde{U}_{1}, \text { out }}^{P D} & =1-\operatorname{Pr}\left(\left\{\left|g_{k}\right|^{2} \geq \beta_{1}\right\}\right)=1-\int_{\max \left(\tau, \beta_{1}\right)}^{\infty} f_{X}(x) d x \\
& =1-\exp \left(-\frac{\max \left(\tau, \beta_{1}\right)}{2 \mu^{2}}\right) .
\end{aligned}
$$

The outage probability of the group 1 users can be found as

$$
\operatorname{Pr}_{U_{1}, \text { out }}^{P D}=\frac{M}{N}\left(\operatorname{Pr}_{\hat{U}_{1}, \text { out }}^{P D}\right)+\frac{N-M}{N}\left(\operatorname{Pr}_{\tilde{U}_{1}, \text { out }}^{P D}\right)
$$


and of the group 2 users as

$$
\begin{aligned}
\operatorname{Pr}_{U_{2}, \text { out }}^{P D} & =1-\rho_{2} \rho_{3} C_{2}\left(e^{-\frac{\beta_{2}}{2 \mu^{2}}}-e^{-\frac{\tau-\beta_{1}}{2 \mu^{2} \alpha}}\right) \\
& -\rho_{2} \gamma e^{-\frac{\beta_{1}}{2 \mu^{2}}}\left(e^{-t_{2} \frac{\alpha+1}{2 \mu^{2}}}-e^{-\frac{(\alpha+1) \tau}{2 \mu^{2}}}\right)
\end{aligned}
$$

by assuming that when $\beta_{2}$ is larger than $\tau$, all $U_{2}$ users are in outage. Further, in order to simplify the integration limits we have defined $t_{2} \triangleq-\max \left(\beta_{2}, \frac{\tau-\beta_{1}}{\alpha}\right), \rho_{1} \triangleq \mathrm{u}\left(\beta_{1}-\tau\right)$, $\rho_{2} \triangleq \mathrm{u}\left(\beta_{2}-\tau\right)$ and $\rho_{3} \triangleq \rho_{2} \mathrm{u}\left(\beta_{2}-\frac{\tau-\beta_{1}}{\alpha}\right)$ where $\mathrm{u}(\cdot)$ is the unit step function.

Then, PD-NOMA outage probability can be expressed as

$$
P r_{\text {out }}^{P D}=\frac{N \operatorname{Pr}_{U_{1}, \text { out }}^{P D}+M P r_{U_{2}, \text { out }}^{P D}}{M+N}
$$

\section{B. NOMA-2000}

As with PD-NOMA, coverage events for user $n \in U_{1}$ will be denoted as $E_{U_{1}, n}^{F D}$. The outage probability for $n \in U_{1}$, $\operatorname{Pr}_{\text {out }}^{F D}(n)=1-\operatorname{Pr}\left(E_{U_{1}, n}^{F D}\right)$, which can be written as

$$
P r_{\text {out }}^{F D}(n)=1-\operatorname{Pr}\left(\left|g_{n}\right|^{2}>\frac{\alpha}{N} \sum_{j=1}^{M}\left|g_{j}\right|^{2}+\beta_{n}\right) \text {. }
$$

Unlike PD-NOMA, the signals of MC-CDMA users can be decoded under the interference caused by the decoding error of OFDMA users. If the signal of the $n$th OFDMA user is incorrectly decoded, it causes an interference with power $4 P_{n}\left|h_{n}\right|^{2} / N$. For the sake of the simplicity, assume that the users in $U_{1}$ are sorted in descending order of their channel gains. Then, define $E_{U_{2}, m, k}^{F D}$ as the event that $m$ th user of $U_{2}$ is on the coverage under the interference caused by $k$ users with lowest channel gains from $U_{1}$. Accordingly, we can write the outage probability of a MC-CDMA user as

$$
\begin{aligned}
P_{\text {out }}^{F D}\left(U_{2}, m\right) & =1-\operatorname{Pr}\left(\bigcup_{k=0}^{N}\left(E_{U_{2}, m, k}^{F D} \cap E_{U_{1}, N-k}^{F D}\right)\right), \\
E_{U_{2}, m, k}^{F D} & =\left\{\left|g_{m}\right|^{2}>\frac{4 \phi}{\epsilon N} \sum_{i=N+1-k}^{N}\left|g_{i}\right|^{2}+\beta_{2}\right\}(.21)
\end{aligned}
$$

For simplicity, we first define a new random variable $Z \triangleq$ $N^{-1} \sum_{k=1}^{M} Y_{k}$ modeling the interference on each subcarrier caused by signals from $U_{2}$. Invoking the central limit theorem, we approximate $Z$ with a Gaussian random variable. Then the following relations hold between the channel gains of $\mathrm{MC}$ CDMA users and interference: $E[Z]=\frac{M}{N} E[Y], E\left[Z^{2}\right]=$ $\frac{M}{N^{2}} E\left[Y^{2}\right]+\frac{M^{2}-M}{N^{2}} E[Y]^{2}, \operatorname{Var}(Z)=\frac{M}{N^{2}} E\left[Y^{2}\right]-\frac{M}{N^{2}} E[Y]^{2}$ where $E[Y]=2 C_{2} \mu^{2}-C_{2} e^{-\frac{\tau}{2 \mu^{2}}}\left(\tau+2 \mu^{2}\right)$ and $E\left[Y^{2}\right]=$ $8 C_{2} \mu^{4}-C_{2} e^{-\frac{\tau}{2 \mu^{2}}}\left(\tau^{2}+4 \tau \mu^{2}+8 \mu^{4}\right)$.

Defining $\mu_{Z} \triangleq E[Z]$ and $\sigma_{Z}^{2} \triangleq \operatorname{Var}(Z)$, the distribution of the interference can be approximated as $Z \sim \mathcal{N}\left(\mu_{Z}, \sigma_{Z}^{2}\right)$. To obtain a closed-form solution, the terms associated with signals decoded under interference are removed from equation (21). With this removal, we assume that if any OFDMA users are in outage, so are all MC-CDMA users regardless of their SNR values. This means that the outage probabilities of MC-CDMA users are bounded by that of the weakest OFDMA user. As in PD-NOMA, we assume that the channel gains of all MCCDMA users are less than $\tau$ and have the PDF of $Y$. Then, the outage probability for MC-CDMA users can be derived as

$$
\begin{aligned}
& \operatorname{Pr}_{U_{2}, \text { out }}^{F D}=1-\operatorname{Pr}\left(Z<\frac{\tau}{\alpha}-\mu_{Z}, Y>\beta_{2}\right) \\
& \approx 1-\operatorname{Pr}\left(Z<\frac{\tau}{\alpha}-\mu_{Z}\right) \operatorname{Pr}\left(Y>\beta_{2}\right) \\
& \quad=1-\rho_{2}\left(1-Q\left(\frac{\frac{\tau-\beta_{1}}{\alpha}-\mu_{Z}}{\sigma_{Z}}\right)\right)\left(1-C_{2}+C_{2} e^{-\frac{\beta_{2}}{2 \mu_{Z}^{2}}}\right),
\end{aligned}
$$

where $Q(\cdot)$ denotes the Gaussian tail function.

The outage probability for $U_{1}$ users can also be derived as

$$
\begin{gathered}
\operatorname{Pr}_{U_{1}, \text { out }}^{F D}=1-\operatorname{Pr}\left(X>\frac{\alpha}{N} Z+\beta_{1}\right) \approx 1-\operatorname{Pr}\left(X>\frac{\alpha}{N} \mu_{Z}+\beta_{1}\right) \\
=1-\int_{\max \left(\frac{\alpha}{N} \mu_{Z}+\beta_{1}, \tau\right)}^{\infty} f_{X}(x) d x=1-C_{1} e^{-\frac{\max \left(\frac{\alpha}{N} \mu_{Z}+\beta_{1}, \tau\right)}{2 \mu^{2}}}
\end{gathered}
$$

by considering $\sigma_{z} \ll \mu_{z}$ and approximating the interference as $\mu_{Z}$. Then, the average outage probability can be written as

$$
\operatorname{Pr}_{\text {out }}^{F D}=\frac{N \operatorname{Pr}_{U_{1}, \text { out }}^{F D}+M \operatorname{Pr}_{U_{2}, \text { out }}^{F D}}{M+N} .
$$

\section{Simulation Results}

In this section we validate our analysis and compare the two NOMA schemes using computer simulations. For all simulations, the power-imbalance parameter $\epsilon$ was selected as 0.5 , and $10^{6}$ iterations were performed.

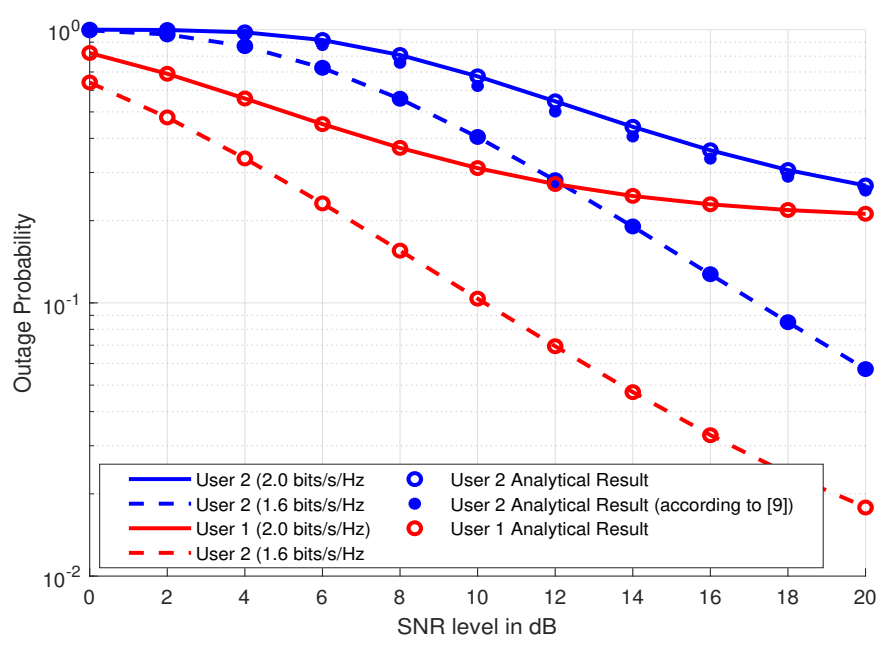

Figure 1: Outage performance of the single carrier case.

Fig. 1 shows the outage probabilities for the single-carrier case. Simulations are repeated for different target rates. It is seen that the simulation results for all users and situations validate equations (14) and (15). On the other hand, we show that there is a significant difference between simulation results and the analytical results in [9] for a target spectral efficiency $2.0 \mathrm{bit} / \mathrm{s} / \mathrm{Hz}$. This difference disappears when the target spectral efficiency is $1.6 \mathrm{bit} / \mathrm{s} / \mathrm{Hz}$, because this value makes the value of $\alpha$ become close to 1 , thus making events $E_{1}$ and $E_{2}$ approximately independent.

For both multicarrier PD-NOMA and NOMA-2000, the simulations are carried out with the same parameters. In these 


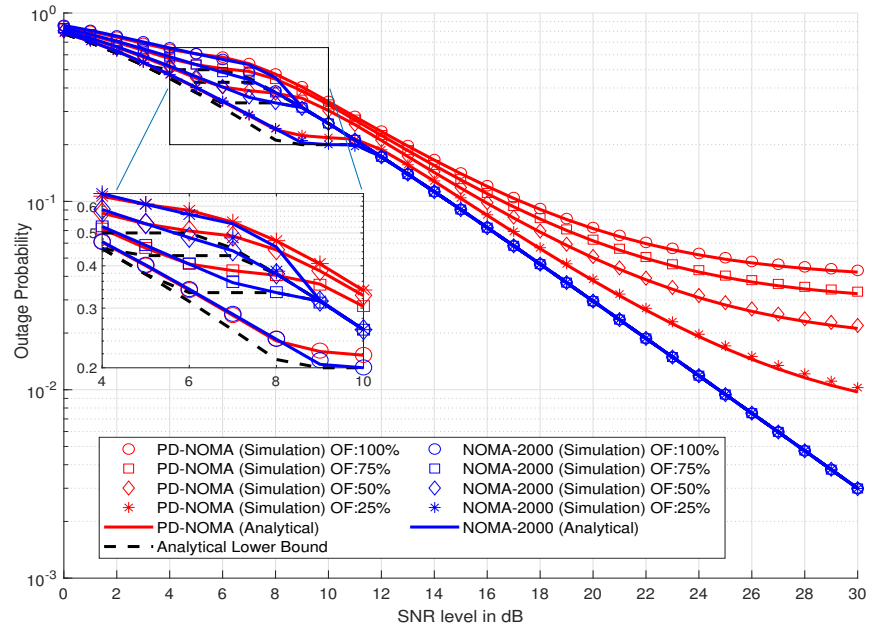

Figure 2: Outage performances of the multi-carrier NOMA systems for $\hat{R}=2$ bits/s/Hz, $\mathrm{N}=256$ subcarriers.

simulations, 256 subcarriers are used and the target spectral efficiency is chosen as $\hat{R}=2 \mathrm{bit} / \mathrm{s} / \mathrm{Hz}$ with different overloading factors. In Fig. 2, it can be seen that the results (19) from the analysis are close to those obtained by the simulations. A small disagreements between (24) and the simulation results can be observed, especially between 7 and $9 \mathrm{~dB}$. For more accuracy, equation (22) may be expanded by considering the situations where MC-CDMA users can decode their signal under the interference caused from weak OFDMA users. Hence, we can conclude that all assumptions are valid for both NOMA schemes and user groups, and for all overloading factors. It can also be seen that the outage probabilities of PD-NOMA reach an error floor at SNR $>15 \mathrm{~dB}$. This is due to the fact that some subcarriers experience relatively higher interference, which limits the performance as the OF increases. On the other hand, in NOMA-2000 the interference occurs due to the average of the received powers of all users $U_{2}$, causing the outage probabilities to decrease dramatically with SNR. At high SNR, outages in $U_{2}$ dominate the performance of NOMA-2000, therefore the performances are similar at different OFs.

In Fig. 2, we also present a theoretical lower bound which corresponds to the orthogonal multiple access (OMA) case where all users are allocated to an orthogonal resource block without sharing it with any other user. It can be derived easily from the NOMA analytical derivations as a special case as $\frac{N}{K} \int_{0}^{\beta_{1}} f_{X}(x) d x+\frac{M}{K} \int_{0}^{\beta_{2}} f_{Y}(y) d y$. Notice from Fig. 2, NOMA-2000 equipped with a simple dynamical user grouping algorithm not only outperforms PD-NOMA but also actually performs very close to this bound at low SNR values where the gap is due to the imperfect SIC at these SNR's and reaches the OMA lower bound at moderate-high SNR regions. We conclude that NOMA-2000 outperforms PD-NOMA under dynamic user grouping. This performance improvement can be justified by the fact that NOMA-2000 inherently exploits the code domain in addition to the power domain as given in [5].

\section{CONCLUSION}

In this paper, exact outage probability expressions have been derived for both PD-NOMA and NOMA-2000 systems in single- and multi-carrier uplink scenarios assuming Rayleigh fading channels. This has been done by taking into consideration the statistical dependencies caused by each resource block possibly being occupied by more than one user signal. Both the analytical derivations and simulation results corroborate the fact that NOMA-2000 significantly outperforms PD-NOMA in terms of outage probability.

Notice that both for clarity of presentation and also for fair comparisons with previous work on the subject, a practical user grouping strategy of [6] and a fixed power allocation strategy have been considered in both the analytical derivations and simulations. However, future work will involve the use of more sophisticated user pairing algorithms such as those proposed in [15], [16], advanced SIC schemes as in [17] and outage optimum power allocation to evaluate and compare the optimum performances of both approaches. Another interesting direction is the extension of this work to other fading channel models and to the analysis under imperfect channel state information (CSI). Future work may also involve the performance analysis and comparison of PD-NOMA and NOMA-2000 in terms of other important performance metrics such as capacity, average BER, fairness and complexity.

\section{REFERENCES}

[1] L. Dai, et. al., "Non-orthogonal multiple access for 5G: solutions, challenges, opportunities, and future research trends," IEEE Communications Magazine, vol. 53, no. 9, pp. 74-81, Sept. 2015

[2] Z. Ding, et. al., "A Survey on non-orthogonal multiple access for 5G networks: Research challenges and future trends," IEEE J. on Select. Areas in Commun., vol. 35, no. 10, pp. 2181-2195, Oct. 2017.

[3] Z. Yang, et. al., "On the optimality of power allocation for NOMA downlinks with individual QoS constraints," IEEE Communications Letters, vol. 21, no. 7, pp. 1649-1652, July 2017.

[4] H. Sari, et. al., "On the foundation of NOMA and its application to 5G cellular networks," Proc. WCNC 2018, April 2018, Barcelona, Spain.

[5] A. Maatouk, et. al., "Frequency-domain NOMA with two sets of orthogonal signal waveforms," IEEE Communications Letters, vol. 22, no. 5, pp. 906-909, May 2018.

[6] E. Caliskan, M. Koca, G. Gui and H. Sari, "Uplink performance of NOMA-2000 with dynamic user grouping," in Proc. IEEE PIMRC 2019, Istanbul, Turkey, pp. 1-6, 2019.

[7] H. Sari, F. Vanhaverbeke, and M. Moeneclaey, "Multiple Access Using Two Sets of Orthogonal Signal Waveforms," IEEE Communications Letters, vol. 4, no. 1, pp. 4-6, Jan. 2000

[8] H. Sari, F. Vanhaverbeke and M. Moeneclaey, "Extending the capacity of multiple access channels," IEEE Communications Magazine, vol. 38, no. 1, pp. 74-82, Jan. 2000.

[9] N. Zhang et al., "Uplink nonorthogonal multiple access in 5G systems", IEEE Communications Letters, vol. 20, no. 3, pp. 458-461, March 2016.

[10] L. Ye, M. Derakhshani, and S. Lambotharan. "Outage analysis and power allocation in uplink non-orthogonal multiple access systems," IEEE Communications Letters vol. 22, no. 2, pp. 336-339, Feb. 2017.

[11] Y. Gao, et. al., "Theoretical Analysis of the Dynamic Decode Ordering SIC Receiver for Uplink NOMA Systems," IEEE Communications Letters, vol. 21, no. 10, pp. 2246-2249, Oct. 2017.

[12] J. Wang, et. al., "Outage Performance Analysis for Wireless NonOrthogonal Multiple Access Systems," IEEE Access, vol. 6, pp. 36113618, 2018.

[13] B. Xia, et. al."Outage Performance Analysis for the Advanced SIC Receiver in Wireless NOMA Systems," IEEE Transactions on Vehicular Technology, vol. 67, no. 7, pp. 6711-6715, July 2018.

[14] 3GPP TR 36.213, V10.12.0, 'Physical layer procedures,' Sept. 2015.

[15] Y. Fu, et. al., "Zero-Forcing Oriented Power Minimization for MultiCell MISO-NOMA Systems: A Joint User Grouping, Beamforming, and Power Control Perspective," IEEE Journal on Selected Areas in Communications, vol. 38, no. 8, pp. 1925-1940, Aug. 2020.

[16] M.A. Sedaghat, R. R. Muller, "On User Pairing in Uplink NOMA," IEEE Trans. Wireless Commun., vol. 17 no. 5, pp. 3474-86, May 2018.

[17] Z. Ding, R. Schober, H. V. Poor, "Unveiling the Importance of SIC in NOMA Systems: Part I -State of the Art and Recent Findings, Part II New Results and Future Directions," IEEE Commun. Letters, 2020. 